\title{
CMEARTICLE
}

\section{Clinics in diagnostic imaging (204)}

Jinhang $\underline{\text { Wen }}^{1}$, FRCR, MMed, Vasu Keshav Sharma ${ }^{2}$, MBBS, FRCR, Aung $\underline{\underline{L} \text { win }^{3}}$, MMed, FRCS, Joey Chan Yiing $\underline{B e h}^{2}$, FRCR, MMed
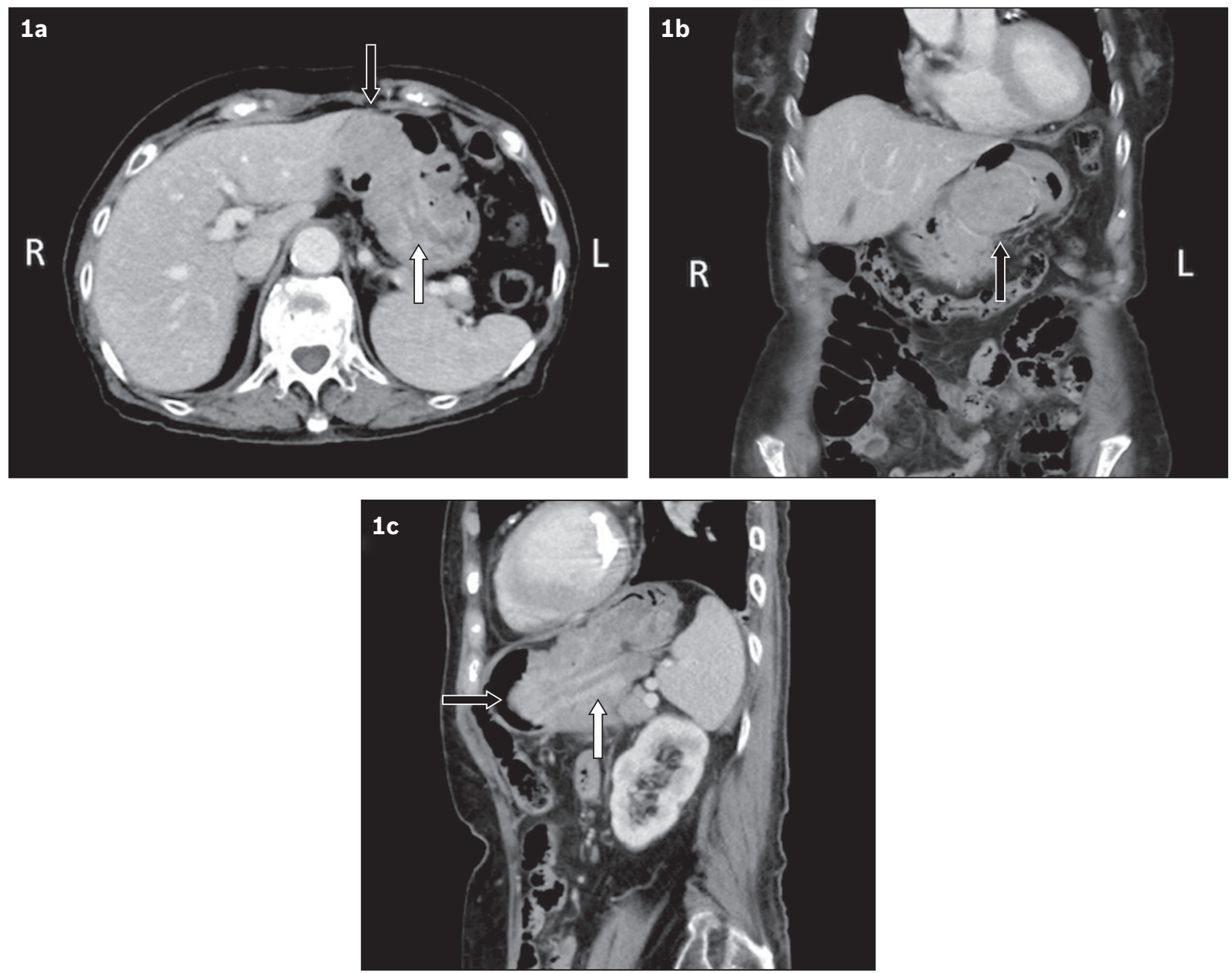

Fig. 1 Contrast-enhanced (a) axial, (b) reconstructed coronal and (c) reconstructed sagittal CT images of the upper abdomen.

\section{CASE PRESENTATION}

A 95-year-old woman with a past medical history of hypertension and chronic kidney disease presented with abdominal bloating and poor appetite of one week's duration. Physical examination showed conjunctival pallor and mild generalised abdominal tenderness without signs of peritonism. Blood tests revealed anaemia of $6.8 \mathrm{~g} / \mathrm{dL}$ from a baseline level of $11.5 \mathrm{~g} / \mathrm{dL}$. Liver function tests and amylase were within normal limits.

Initial chest and abdominal radiographs were unremarkable. Contrast-enhanced computed tomography (CT) of the abdomen and pelvis (Fig. 1) was subsequently performed. What do the images show? What is the diagnosis?

${ }^{1}$ Department of Diagnostic Imaging, National University Hospital, ${ }^{2}$ Department of Diagnostic Imaging, ${ }^{3}$ Department of General Surgery, Ng Teng Fong General Hospital, Singapore Correspondence: Dr Jinhang Wen, Resident, Department of Diagnostic Imaging, National University Hospital, 5 Lower Kent Ridge Road, Singapore 119074. jinhang_wen@nuhs.edu.sg 

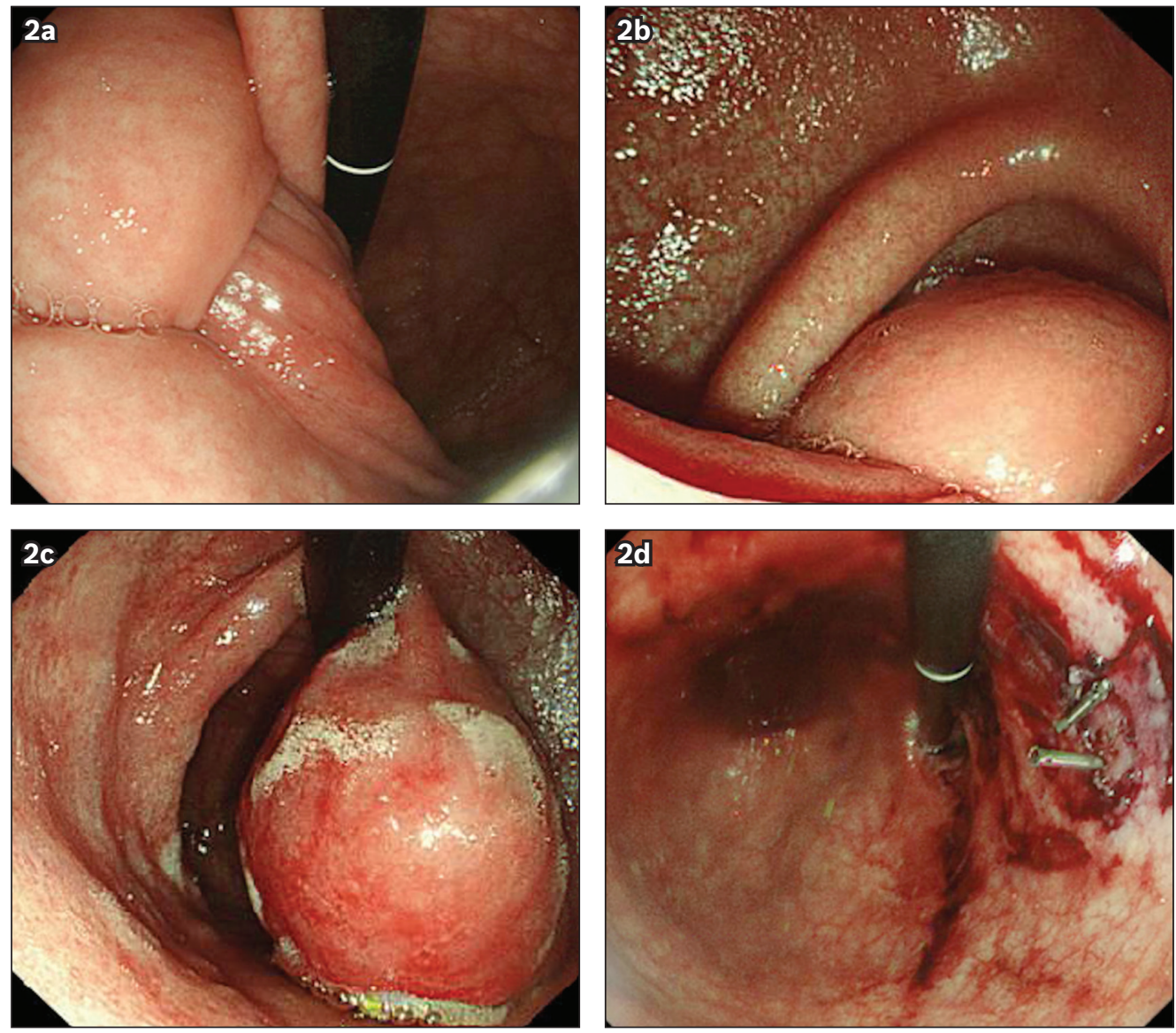

Fig. 2 Oesophagogastroduodenoscopic images show (a) gastro-gastric intussusception; (b) a polyp from the fundus; (c) the polyp after reduction; and (d) post excision.

\section{IMAGE INTERPRETATION}

Contrast-enhanced CT images of the abdomen and pelvis show a well-defined, heterogeneously enhancing intraluminal gastric mass, measuring $5.5 \mathrm{~cm} \times 3.8 \mathrm{~cm}$, infiltrating the wall of the gastric fundus (black arrows, Fig. 1). The stomach is of abnormal configuration with a 'twisted' and distorted appearance, resulting in a medial shift of the gastroduodenal junction. Axial and sagittal reconstructions show a bowel-within-bowel configuration and a 'soft tissue sausage' appearance (white arrows, Fig. 1). These findings are typical of gastro-gastric intussusception with a lead mass. No definite perforation, ischaemia or lymphadenopathy is detected.

\section{DIAGNOSIS}

Gastro-gastric intussusception due to gastric gastrointestinal stromal tumour (GIST).

\section{CLINICAL COURSE}

The patient subsequently underwent oesophagogastroduodenoscopy, which confirmed gastro-gastric intussusception (Figs. 2a \& b). After the intussusception was reduced with pneumoinsufflation, a $5-\mathrm{cm}$ pedunculated polyp was visualised arising from the gastric fundus just distal to the cardia (Fig. 2c), with ulceration and slough at its tip. Pangastritis and inflammation of the first duodenal segment were also noted. No other source of bleeding was seen.
In view of the large size of the polyp and the patient's relatively good premorbid status, the decision was made to perform endoscopic polypectomy with open gastrostomy. On endoscopic polypectomy, recurrence of gastro-gastric intussusception was noted (Fig. 2a). The intussusception was reduced and the polyp was excised at the base of the peduncle (Fig. 2d). Histopathology of the specimen revealed a GIST that was $5.0 \mathrm{~cm}$ in size with a mitotic rate of up to four mitoses per 50 high-power fields. The patient recovered from the operation uneventfully and was discharged well on Postoperative Day 4.

\section{DISCUSSION}

Intussusception refers to the invagination of one part of the gastrointestinal tract into another, most commonly a proximal segment of bowel (intussusceptum) into a distal segment (intussuscipiens). Intussusceptions have been classified into four categories depending on their location: (a) entero-enteric, involving the small bowel only; (b) ileo-colic, with prolapse of the terminal ileum into the ascending colon and beyond; (c) colo-colic, involving the large bowel only; and (d) sigmoidorectal, involving the sigmoid colon and rectum. The enteroenteric type is the most common, followed by the ileo-colic, colo-colic and sigmoido-rectal types. Foregut involvement, as described in the case above, is exceedingly rare and is not part of any anatomical classification mentioned in our review of the literature. 

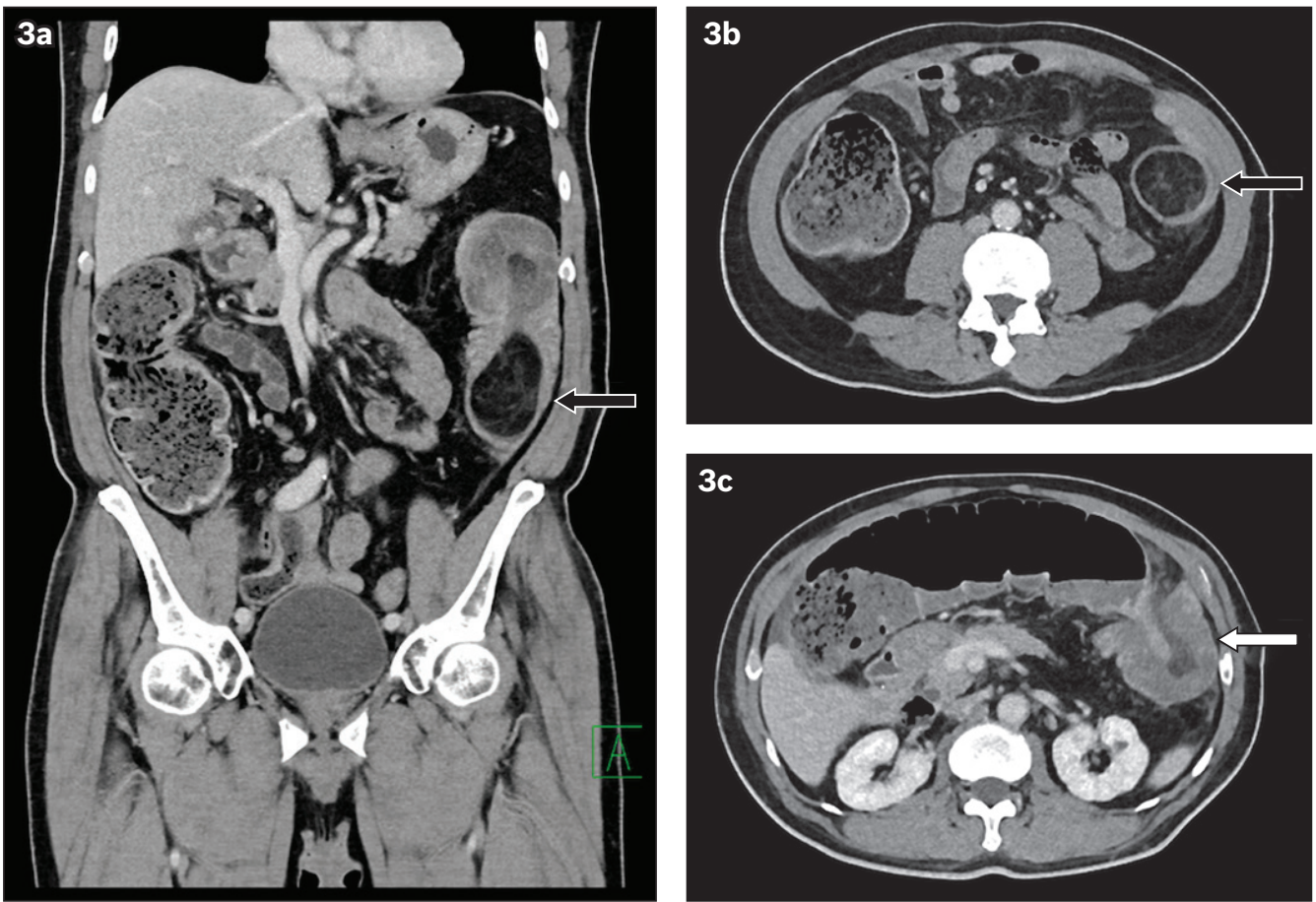

Fig. 3 (a) Coronal and (b \& c) axial CT mesenteric angiograms of a 70-year-old man show a predominantly fatty mass in the descending colon lumen (black arrows). Proximal colonic intussusception is noted, with the entry point at the level of the splenic flexure (white arrow). Following left hemicolectomy and omentectomy, histology revealed a submucosal lipoma.

Gastro-gastric intussusception results from the invagination of the proximal stomach into the distal stomach. There have only been several published cases of gastro-gastric intussusception, ${ }^{(1-6)}$ all of which have a gastric tumour as a lead point. The gastric tumours in question include primary gastric lymphoma, ${ }^{(1)}$ gastric carcinoma $^{(2)}$ and hypertrophic gastric polyps. ${ }^{(3)}$ Only two cases of gastro-gastric intussusceptions due to leiomyomas ${ }^{(4,5)}$ and one due to a GIST ${ }^{(6)}$ have previously been reported.

Adult intussusception is much more rare than its paediatric counterpart, accounting for only $5 \%-10 \%$ of all cases of intusssuceptions ${ }^{(6-8)}$ and $1 \%-5 \%$ of bowel obstruction in adults. ${ }^{(7)}$ In contrast to paediatric intussusception, which classically presents with the triad of abdominal pain, mass and per-rectal bleeding, the clinical presentation of intussusception in adults is usually non-specific. The most common presenting symptoms include abdominal pain, nausea and emesis in the acute setting, and intermittent abdominal pain and vomiting in the subacute or chronic setting. ${ }^{(9)}$ Given the often-vague presenting symptoms, imaging investigations are of value in the evaluation of adult patients with suspected intussusception.

Abdominal radiographs, which are typically the first imaging investigation obtained, may be useful in demonstrating signs of intestinal obstruction and providing information regarding the site of obstruction. Contrast fluoroscopic studies, namely upper gastrointestinal contrast series for foregut/small bowel involvement and barium enema for large bowel involvement, may show a classic 'coiled spring' appearance. ${ }^{(4,5)}$

Ultrasonography is a frequently employed modality for diagnosing intussusception, especially in the paediatric population, although it is also used in adults. Classic sonographic features include the 'target' sign on the transverse view and the 'pseudo-kidney' sign on the longitudinal view. ${ }^{(7)}$ The limitations of ultrasonography include its operator dependency and certain patient factors such as gaseous bowel distension, which may limit image quality and diagnostic accuracy. ${ }^{(7)}$

$\mathrm{CT}$ is currently considered the most useful and sensitive modality to diagnose intussusception in adults, with a reported diagnostic accuracy of $58 \%-100 \%{ }^{(7,8,10,11)}$ Characteristic CT features are well documented, such as the 'target' or 'sausage' sign and invagination of mesenteric fat and vessels within the bowel lumen. ${ }^{(7)}$ Unlike ultrasonography, CT is not affected by the presence of gas in the bowel. ${ }^{(8)} \mathrm{CT}$ can also help to define the location and nature of the lead mass and its relationship to the surrounding tissues. ${ }^{(8,12)}$ In addition, other findings such as the presence of lymphadenopathy or metastasis may also be detected in the setting of an underlying malignancy. ${ }^{(7,8)}$

Adult intussusception is usually caused by a structural lesion acting as a lead mass, ${ }^{(6-8)}$ as opposed to intussusception in children, which is usually primary and benign. ${ }^{(7)}$ It is believed that the presence of a lesion in the wall of the gastrointestinal tract alters normal peristaltic activity, initiating the invagination, with subsequent peristaltic activity causing further telescoping of the lead point. ${ }^{(8-11)}$

One of the most common benign lead masses in adult intussusception is a lipoma (Fig. 3). 90\% of gastrointestinal lipomas are within the submucosa, are usually solitary, and may be sessile or pedunculated. ${ }^{(12)}$ They can be easily diagnosed on CT due to their typical fatty attenuation. It is also not uncommon for an underlying primary malignancy, most frequently colonic adenocarcinoma, to initially present as an intussusception (Fig. 4).

Underlying gastrointestinal polyposis syndromes must be considered when an adult patient presents with suggestive clinical features in addition to intussusception (Figs. 5a \& b). Peutz-Jeghers syndrome is a rare autosomal dominant inherited 

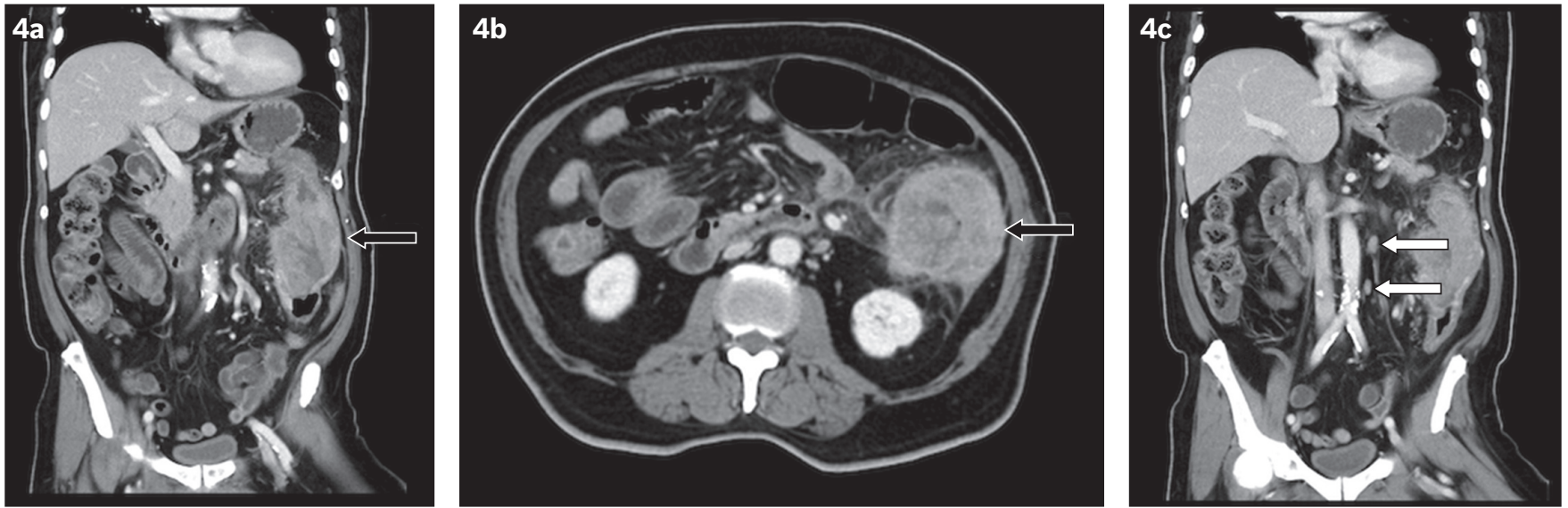

Fig. 4 (a \& c) Coronal and (b) axial contrast-enhanced CT images of the abdomen and pelvis of an 84-year-old man show a long segment of diffuse irregular thickening along the descending colon with colocolic intussusception (black arrows). Enlarged para-aortic lymph nodes are also seen (white arrows). Following subtotal colectomy, histology revealed moderately differentiated adenocarcinoma.
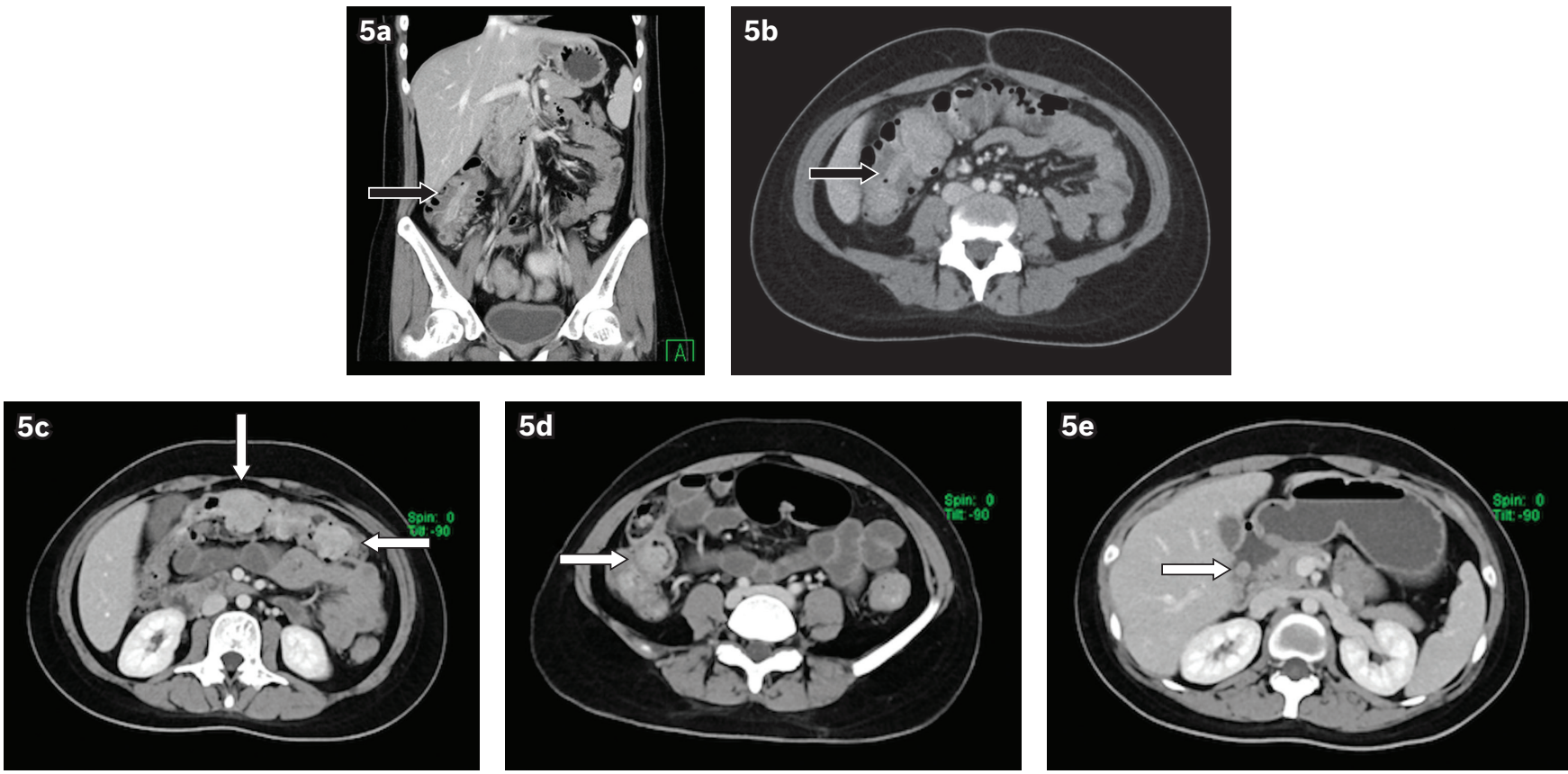

Fig. 5 (a) Coronal and (b) axial contrast-enhanced CT abdomen and pelvis of a 25-year-old woman shows a bowel-within-bowel configuration in the ascending colon, raising suspicion of an ileo-colic intussusception (black arrows). (c-e) Subsequent axial contrast-enhanced CT enterography shows multiple polypoidal lesions in the small and large bowel loops (white arrows), in keeping with the hamartomatous polyps of Peutz-Jeghers syndrome. The patient underwent subtotal colectomy with jejunal and duodenal enterotomy for polypectomy.

disease that is characterised by mucocutaneous pigmentation and gastrointestinal hamartomatous polyps. The sites most commonly affected by polyps are the jejunum, colorectal region, duodenum and stomach, in decreasing order of frequency. Small bowel polyps causing recurrent intussusception is one of the major causes of morbidity in this condition. ${ }^{(13)}$ Patients are also predisposed to various malignancies such as gastrointestinal, pancreatic, lung, breast, uterine, ovarian and testicular tumours.

In conclusion, intussusception in adults is difficult to diagnose preoperatively because it usually presents with nonspecific symptoms, as in this case. A high index of clinical suspicion may be required. The characteristic features of intussusception on CT makes CT a good modality for the evaluation and confirmation of intussusception, including the involvement of unusual sites such as the foregut, as well as for identifying lead masses and excluding other sinister pathology.
ABSTRACT A 95-year-old woman presented with abdominal bloating and anorexia of one week's duration. Contrast-enhanced computed tomography (CT) revealed features in keeping with gastro-gastric intussusception with a lead mass. Oesophagogastroduodenoscopy demonstrated a large $5-\mathrm{cm}$ pedunculated fundal mass intussuscepted into the distal stomach. The patient subsequently underwent endoscopic polypectomy and open gastrostomy. Histological evaluation of the gastric mass revealed a gastrointestinal stromal tumour. Her postoperative course was uneventful. We herein describe the radiological features of adult intussusception and illustrate the usefulness of CT in the detection and characterisation of lead masses.

Keywords: bowel obstruction, computed tomography, gastrogastric, gastrointestinal stromal tumour, intussusception 


\section{REFERENCES}

1. Jo HH, Kang SM, Kim SH, et al. [A case of gastro-gastric intussusception secondary to primary gastric lymphoma]. Korean J Gastroenterol 2016; 68:40-4. Korean.

2. Eom BW, Ryu KW, Lee JH, et al. Gastrogastric intussusception secondary to a gastric carcinoma: report of a case. Surg Today 2011; 41:1424-7.

3. Vikram R, McCulloch AS, Zealley IA. True gastrogastric intussusception: a rare radiologic diagnosis with surgical correlation. AJR Am J Roentgenol 2006; 186:585-6.

4. Javors BR, Rackson M. Gastrogastric intussusception. J Clin Gastroenterol 1987; 9:113-4.

5. Grundy A, Rayter Z, Shorthouse AJ. Gastrogastric intussuscepting leiomyomas. Gastrointest Radiol 1984; 9:319-21.

6. Shanbhogue A, Walsh C, Fasih N. Education and imaging. Gastrointestinal: gastrogastric intussusception. J Gastroenterol Hepatol 2009; 24:169.
7. Marinis A, Yiallourou A, Samanides L, et al. Intussusception of the bowel in adults: a review. World J Gastroenterol 2009; 15:407-11.

8. Wang N, Cui XY, Liu Y, et al. Adult intussusception: a retrospective review of 41 cases. World J Gastroenterol 2009; 15:3303-8.

9. Zubaidi A, Al-Saif F, Silverman R. Adult intussusception: a retrospective review. Dis Colon Rectum 2006; 49:1546-51.

10. Wang LT, Wu CC, Yu JC, et al. Clinical entity and treatment strategies for adult intussusceptions: 20 years' experience. Dis Colon Rectum 2007; 50:1941-9.

11. Goh BK, Quah HM, Chow PK, et al. Predictive factors of malignancy in adults with intussusception. World J Surg 2006; 30:1300-4.

12. Agrawal A, Singh KJ. Symptomatic intestinal lipomas: our experience. Med J Armed Forces India 2011; 67:374-6.

13. Kopacova M, Tacheci I, Rejchrt S, Bures J. Peutz-Jeghers syndrome: diagnostic and therapeutic approach. World J Gastroenterol 2009; 15:5397-408. 


\section{SINGAPORE MEDICAL COUNCIL CATEGORY 3B CME PROGRAMME}

\section{(Code SMJ 202002B)}

Question 1. The following are possible clinical presentations of bowel intussusception:

(a) Acute abdominal pain

(b) Intermittent abdominal pain and vomiting.

(c) Abdominal mass.

(d) Per-rectal bleeding.

Question 2. Regarding lead masses in bowel intussusception:

(a) Intussusception in children is usually due to the presence of a lead mass.

(b) Gastrointestinal lipomas are easily characterised on CT due to their fatty attenuation.

(c) Small bowel polyps in Peutz-Jeghers syndrome predispose a patient to recurrent intussusceptions.

(d) Colonic adenocarcinoma is the most common malignant lead mass.

Question 3. Regarding the use of computed tomography (CT) in the diagnosis of suspected intussusception:

(a) CT is considered the most useful and sensitive modality to diagnose intussusception in children.

(b) Unlike ultrasonography, assessment of intussusception on CT is not affected by the presence of gas in the bowel.

(c) Besides the presence of intussusception, CT can also detect associated findings such as lymphadenopathy or metastasis.

(d) CT is not useful in characterising the nature of the lead mass.

Question 4. The following are classic imaging signs seen in bowel intussusception:

(a) Coiled spring appearance.

(b) Target sign.

(c) Pseudo-kidney sign.

(d) Windsock sign.

Question 5. Regarding the diagnosis of adult intussusception:

(a) A high index of suspicion is required.

(b) Adult intussusception is difficult to diagnose preoperatively as the symptoms may be vague.

(c) CT is typically the first imaging modality obtained.

(d) Abdominal radiographs are of no value in the diagnosis of adult intussusception.

True

False

$\square$

$\square$

$\square$

$\square$

$\begin{array}{ll}\square & \square \\ \square & \square \\ \square & \square \\ \square & \square\end{array}$

\section{Doctor's particulars:}

Name in full:

Specialty:
MCR no.:

Email:

\section{SUBMISSION INSTRUCTIONS:}

Visit the SMJ website: http://www.smj.org.sg/current-issue and select the appropriate quiz. You will be redirected to the SMA login page.

For SMA member: (1) Log in with your username and password (if you do not know your password, please click on 'Forgot your password?'). (2) Select your answers for each quiz and click 'Submit'.

For non-SMA member: (1) Create an SMJ CME account, or log in with your SMJ CME username and password (for returning users). (2) Make payment of SGD 21.40 (inclusive of $7 \%$ GST) via PayPal to access this month's quizzes. (3) Select your answers for each quiz and click 'Submit'.

RESULTS:

(1) Answers will be published online in the SMJ April 2020 issue. (2) The MCR numbers of successful candidates will be posted online at the SMJ website by 10 April 2020. (3) Passing mark is $60 \%$. No mark will be deducted for incorrect answers. (4) The SMJ editorial office will submit the list of successful candidates to the Singapore Medical Council. (5) One CME point is awarded for successful candidates. (6) SMC credits CME points according to the month of publication of the CME article (i.e. points awarded for a quiz published in the February 2020 issue will be credited for the month of February 2020, even if the deadline is in April 2020).

Deadline for submission (February 2020 SMJ 3B CME programme): 12 noon, 3 April 2020. 